\section{Should we be pursuing the earlier diagnosis of lung cancer in symptomatic patients?}

\author{
Michael D Peake
}

Three papers published in this issue of Thorax address the issue of the late diagnosis of lung cancer. ${ }^{1-3}$ The most recent international population-based survival data for lung cancer again show the UK at the bottom of the scale with a predicted $8.8 \%$ of those diagnosed between 2005 and 2007 being alive at 5 years compared with $14.4 \%$ in Norway, $16.3 \%$ in Sweden and $15-20 \%$ in areas of Australia and Canada. ${ }^{4}$ It has been estimated that, in England, around 1300 lives per year would be saved if our survival matched the best in Europe. ${ }^{5}$ It is well known that late diagnosis is the major reason why lung cancer outcomes are generally so poor and this is illustrated by the fact that, in the UK, more than $70 \%$ of patients have advanced stage (IIIB or IV) disease at the time of diagnosis. ${ }^{6}$ Huge attention has therefore been focused on the possibility of screening asymptomatic individuals and the most recent findings of the National Lung Cancer Screening Trial ${ }^{7}$ would appear promising.

In considering reasons underlying the consistent finding of poor UK survival rates, most attention has been given to shortcomings in treatment in secondary care and there is no doubt that there are wide variations in treatment and survival within the UK itself. ${ }^{6}$ What is not yet known is the extent to which later stage at diagnosis in the UK may explain these survival differences. Some have proposed that at least part of the explanation may be a higher rate of comorbidities in the $\mathrm{UK}^{8}$ although the evidence for this is limited. One recent study, however, suggests that late diagnosis in the UK is a major factor. Holmberg et al ${ }^{9}$ compared excess lung cancer deaths at 5 years in different age groups in Sweden, Norway and England. There were considerably more excess deaths in all age groups in England but when patients who died

Correspondence to Dr Michael D Peake, Consultant and Senior Lecturer in Respiratory Medicine, Glenfield Hospital, Leicester National Clinical Lead for NHS Cancer Improvement and the National Cancer Intelligence Network, Groby Road, Leicester LE3 9QP, UK; mick.peake@uhl-tr.nhs.uk within the first year were excluded the death rates among the countries were similar; indeed most of the excess deaths in England could be explained by deaths in the first 3 months after diagnosis. Since there are no screening programmes in any of these countries, the assumption has to be that symptomatic patients are, for whatever reason, getting treatment earlier in Norway and Sweden than in England. That very late diagnosis is an important issue is well illustrated in work by the National Cancer Intelligence Network. This work showed that in 2007, 38\% of all patients with lung cancer in England first presented to secondary care as emergencies. Only $8.9 \%$ of these patients were alive at 1 year compared with $39.8 \%$ of those referred by an elective route from primary care. ${ }^{10}$ Several studies have described delays of many weeks or months in patients seeking medical advice for the symptoms of lung cancer. ${ }^{11} 12$

Simon et $a l^{1}$ developed a robust lung cancer awareness measure and have clearly demonstrated that awareness of the symptoms of, and risk factors for, lung cancer is very poor in the UK, with $38 \%$ of the large study sample unable to recall a single symptom. This cancer awareness measure is now being used as a tool in UKbased public awareness campaigns and in an International Cancer Benchmarking Study to examine international differences in public awareness of symptoms. Athey et $a l^{2}$ report the impact of a campaign in Doncaster to raise public and primary care awareness of lung cancer. The message of the campaign was that a persistent cough should trigger people to seek advice from their GP and to ask for a chest x-ray. This innovative project, which is a collaboration of public health, secondary and primary care clinicians, used social marketing techniques to identify and target communities at high risk of developing lung cancer. One particularly effective method adopted by the campaign was to install chips in bus stops so that the sound of coughing was triggered as people approached. This led to wide local press coverage. Although this has to be considered a pilot project, it clearly showed that the campaign resulted in enhanced public recall of cough as an important symptom; a change in behaviour of the public, with more patients going to their GP; a change in behaviour of GPs, with a $20 \%$ increase in chest $x$-ray requests; and an increase in new lung cancer diagnoses, with a suggestion that there were more patients diagnosed with early stage disease. Clearly further work is needed on a larger scale to confirm these findings and to prove that such an approach can have an impact on radical treatment rates and survival. As part of the government's National Awareness and Early Diagnosis Initiative (NAEDI), a joint project between the English Department of Health and Cancer Research UK, ${ }^{13}$ these findings, taken together with other evidence of late diagnosis referred to above, have led to a number of local public and primary care lung cancer awareness projects and a major public awareness campaign in the Midlands in autumn 2011. This campaign, part of a wider 'Be Clear on Cancer' programme, aims to raise awareness of the symptom of a cough lasting for more than 3 weeks and uses TV, radio, press, etc to convey this message. We await the evaluation of these projects with interest.

Coming back to the screening of asymptomatic people, most international efforts aimed at improving early diagnosis of lung cancer have been driven by the often unspoken belief that, by the time patients with lung cancer develop symptoms, the die is cast and the likely long term survival benefits will be small. All of the major recent work on screening has used low-dose CT scanning. Widely divergent, indeed polarised, views have been expressed by a variety of authors on the relative benefits and cost effectiveness of this approach. ${ }^{14}{ }^{15}$ One issue which is much less discussed, but that may limit the effectiveness of any screening programme, is whether people at high risk would wish to take part in such programmes. ${ }^{16}$ This is the subject of the study by Patel et $a l^{3}$ in the context of the Lung-SEARCH trial. The risks of smoking are so well known that the residual smokers (around $21 \%$ of adults in the $\mathrm{UK}^{17}$ ) are probably people with a pattern of high-risk behaviour with a mind set of fatalism and denial who intuitively one would expect to be a group less likely to take up the offer of screening. There is also a strong culture of nihilism around the diagnosis of lung cancer, which is popularly thought of as a universal death penalty. One previous study has added weight to the idea that poor uptake in this 
group may be an issue, demonstrating that smokers are less likely to participate in lung cancer screening programmes than ex-smokers. ${ }^{18}$ Patel et $a l^{3}$ reported on the acceptance of annual CT scans, sputum cytometry and bronchoscopy surveillance in smokers and ex-smokers with mild to moderate chronic obstructive pulmonary disease (COPD) who had already consented to take part in the LungSEARCH trial. Although the majority of participants found the interventions acceptable, there was evidence of doubt about the value of early detection, particularly in older patients. They also found evidence to support the hypothesis that worry is a factor that deters people from participating in screening. Such considerations clearly need to be built in to costeffectiveness analyses of any national screening programme.

While it is vital we continue to push for effective screening programmes, those that use current technology are likely to be of limited benefit, costly and not likely to be implemented in the near future, at least in the UK. Strenuous efforts are also needed to find better screening methods that can eventually supersede CT scanning. We simply cannot wait and have to trial-and carefully evaluate- - a variety of approaches to promote earlier diagnosis in patients who already have symptoms of lung cancer.
Competing interests MDP is a co-author on the paper by Simon et al, though his contribution was limited to advice on some elements of the text and the conclusions rather than the design or analysis of the study. He is also a participant in the LungSEARCH trial which is the subject of the paper by Patel et al, though he is not a co-author and has not been directly involved in this sub-study or the preparation of the paper. He is the secondary care lead for the NAEDI programme.

Provenance and peer review Commissioned; internally peer reviewed.

Published Online First 17 March 2012

Thorax 2012;67:379-380

doi:10.1136/thoraxjnl-2011-201449

\section{REFERENCES}

1. Simon A, Juszczyk D, Smyth N, et al. Knowledge of lung cancer symptoms and risk factors in the UK: development of a measure and results from a population-based survey. Thorax 2012;67:426-32.

2. Athey V, Suckling R, Tod A, et al. Early diagnosis of lung cancer: evaluation of a community-based social marketing intervention. Thorax 2012;67:412-7.

3. Patel D, Akporobaro A, Chinyanganya N, et al Attitudes to participation in a lung cancer screening trial: a qualitative study. Thorax 2012;67:418-25.

4. Coleman MP, Forman D, Bryant $\mathrm{H}$, et al. Cancer survival in Australia, Canada, Denmark, Norway, Sweden, and the UK, 1995-2007 (the International Cancer Benchmarking Partnership): an analysis of population-based cancer registry data. Lancet 2011;377:127-38

5. Abdel-Rahman $\mathbf{M}$, Stockton D, Rachet B, et al. What if cancer survival in Britain were the same as in Europe: how many deaths are avoidable? Br J Cancer 2009;101(Suppl 2):S115-24.

6. NHS Information Centre. National Lung Cancer Audit. 2010 Annual report. http://www.ic.nhs.uk/ webfiles/Services/NCASP/audits\%20and\%20reports/
NHSIC_National_Lung_Cancer_Audit_2010_V1.0.pdf (accessed 13 Dec 2011).

7. The National Lung Screening Trial Research Team. Reduced lung-cancer mortality with low-dose computed tomographic screening. $N$ Engl J Med 2011;365:395-409.

8. Imperatori A, Harrison $\mathrm{R}$, Leitch $\mathrm{DH}$, et al. Lung cancer in Teesside (UK) and in Varese (Italy): a comparison of management and survival. Thorax 2006;61:232-9.

9. Holmberg L, Sandin F, Bray F, et al. National comparisons of lung cancer survival in England, Norway and Sweden 2001-2004: differences occur early in follow-up. Thorax 2010;65:436-41.

10. National Cancer Intelligence Network. Routes to Diagnosis. NCIN report available via: http://www. ncin.org.uk (accessed 13 Dec 2011).

11. Corner J, Hopkinson J, Fitzsimmons D, et al. Is late diagnosis of lung cancer inevitable? Interview study of patients' recollections of symptoms before diagnosis. Thorax 2005;60:314-19.

12. Jensen AR, Mainz J, Overgaard J. Impact of delay on diagnosis and treatment of primary lung cancer. Acta Oncol 2002:41:147-52.

13. Cancer Research UK: About NAEDI. http://info. cancerresearchuk.org/spotcancerearly/naedi/ AboutNAEDI/ (accessed 13 Dec 2011).

14. Gleeson FV. Screening for lung cancer with spiral CT is not justified. Nat Clin Pract Oncol 2007;4:442-3.

15. Henschke C. CT screening for lung cancer is justified. Nat Clin Pract Oncol 2007:4:440-1.

16. Spiro SG. Screening for lung cancer: yet another problem. Thorax 2007;62:105-6.

17. Office for National Statistics. General Lifestyles Survey (Data for 2009). http://www.ons.gov.uk/ons/ publications/re-reference-tables.html?edition $=\mathrm{tcm} \%$ 3A77-198839 (accessed 16 Dec 2011).

18. Silvestri GA, Nietert PJ, Zoller J, et al. Attitudes towards screening for lung cancer amongst smokers and their non-smoking counterparts. Thorax 2007;62:126-30.

\section{Airway disease and emphysema on CT: not just phenotypes of lung pathology}

\section{Peter D Paré, ${ }^{1}$ Pat G Camp}

In this issue of the journal, Martinez et al ${ }^{1}$ examined the relationships between quantitative CT (OCT) parameters of emphy-

\footnotetext{
1Department of Medicine, Camp Department of Physical Therapy, University of British Columbia, James Hogg Research Centre, St. Paul's Hospital, Vancouver, Canada; ${ }^{2}$ Department of Physical Therapy, University of British Columbia, James Hogg Research Centre, St Paul's Hospital, Vancouver, Canada

Correspondence to Dr Peter D Paré, Department of Medicine, Camp Department of Physical Therapy, James Hogg Research Center, St. Paul's Hospital, University of British Columbia, 1081 Burrard Street, Vancouver V6Z 1Y6, Canada; peter.pare@hli.ubc.ca
}

sema, airway wall remodelling and airway narrowing and composite clinical and physiological indices of chronic obstructive pulmonary disease (COPD), the BODE index ${ }^{2}$ and the St George's Respiratory Questionnaire (SGRQ). ${ }^{3}$ BODE stands for Body mass index (BMI), airflow Obstruction, Dyspnoea and Exercise capacity.

Not surprisingly, these OCT estimates of pathological changes were related to measures of clinical impact. More interestingly, the authors found that there were differences in the strength of the associations between measures of emphysema and airway disease and the composite indices. Measures of emphysema were more closely related with the BODE index while the airway wall abnormalities were better predictors of the SGRO.

While it has long been recognised that there is a spectrum of changes in the airways and parenchyma in $\mathrm{COPD},{ }^{4}$ the separation of the airway predominant phenotype from the parenchymal predominant phenotype was largely limited to the autopsy room until the advent of CT. CT has confirmed that some patients have airflow obstruction with little emphysema while others have predominant emphysema with little airway disease. Such individuals form the extremes while the majority of patients have various combinations of airway disease and emphysema. ${ }^{5}$ In addition, there is evidence that the predominant pattern is to some extent familial ${ }^{6}$ and is associated with different rates of decline of lung function. ${ }^{7}$ The presence of airway disease and emphysema on CT can be assessed qualitatively or quantitatively. The power 